\title{
The Vocal Range of Movies - Sonifying Gender Representation in Film
}

\author{
Marcello A. Gómez Maureira and Lisa E. Rombout \\ LIACS Media Technology, Leiden University, \\ Niels Bohrweg 1, Leiden, Netherlands \\ http://mediatechnology. leiden.edu
}

\begin{abstract}
Research has shown that in contemporary movies, male characters consistently outnumber female characters. In recent years, the number of speaking roles identified as female has declined or remained stable. Guidelines like the Bechdel and Mako Mori test have emerged as a method of evaluating gender representation in film. In this study, a more abstract and experiential form of evaluation is proposed. The per-segment sonification of the assigned gender of a character and the amount of lines they have in that segment of the script creates an audio file, showcasing the gender-representation in the movie dynamically. Two focus groups, one specifically consisting of young filmmakers, have expressed their interest in this form of movie-sonification. Expressed wishes for additional features and other suggested improvements are taken into consideration for the creation of the next prototype.
\end{abstract}

Keywords: Gender; Sonification; Representation; Data Perceptualization; Movie; Film; Audio

\section{Introduction}

In a changing landscape of cultural norms, popular media such as movies can reflect as well as influence the current values of society. Trend-analysis of popular movies can therefore offer insightful information about these norms and values. In 2012, Bleakley et al. [1] presented an analysis of the 855 top-grossing films from 1950 to 2006. They concluded that for the main roles, male characters consistently outnumbered female characters by two to one. Female characters where further increasingly likely to be involved in explicit sexual content, and both genders saw a rise in involvement with violent content. A year later, Smith et al. [2] concluded that in the 100 most successful films in 2012, just 28.4 percent of the 4475 speaking characters were female. In 2009, this was 32.8 percent. The amount of speaking lines is, for most films, a representative measure for the importance of a character. Behind the camera, a similar gender-representation pattern emerges, with only one in five directors, producers or writers being women - a figure that has remained stable over time. This suggests that there is a link between gender-representation behind and in front of the camera. 


\subsection{Existing Evaluation Methods}

The Bechdel test (see Fig. 1a) famously checks whether 1) there are at least two female characters in the movie, who 2) have a conversation with each other, about 3) something other than a man. One estimation is that at least $40 \%$ of popular movies do not pass it [3]. From a self-described "little joke" it has become a sort of industry standard to bring the representation of women in cinema to attention. However, it also has several drawbacks. It is hard to give an objective measure: what counts as a character and a conversation is not defined. It does not work for movies with very few characters or dialogue, as these often fail the test automatically. This last problem led to the creation of the similar Mako Mori test [4], which a movie passes if it has 1) at least one female character, 2) who has her own narrative arc, 3) that is not about supporting a man's story.

As both the Bechdel and similar tests works in terms of 'passing' them and rules that need to be adhered to, they are often misunderstood as a verdict on whether a movie is sexist or not. However, while passing the Bechdel test is certainly an indication of a certain gender representation, is does not necessarily say anything about gender depiction.

\subsection{Sonification of Gender}

In this study we are interested in devising a method to intuitively grasp the gender balance in a movie, without a rule system that the movie needs to 'pass' or an otherwise seemingly objective measure of sexism. The goal is to induce thought and discussion about gender representation in movies by separating it from the rest of the movie to be viewed on its own. We decided to focus on the amount of speaking lines as a good benchmark for a characters importance in the movie.

\section{Methods}

\subsection{The Sonification Process}

The very first prototype sonifications were made by analyzing the scripts by hand. A list of five main characters was determined by amount of speaking lines. Each was assigned a different pitch. The scripts were divided into segments, and in each section the number of words spoken by each character was measured. This data was then used to create a sonification, using the Sonification Sandbox [5] an application that allows the mapping of data to sound.

The resulting sonification goes through the segments in order, with tones signifying that a character speaks and volume being dependent on the amount of words they have in that section. Scene descriptions are also represented and result in silent parts whenever the selected five characters do not have lines. Thus, action-heavy movies tend to have more stretches of silence than movies that feature a lot of dialogue. The results were three audio-files of radically different movies ('American Beauty', 'Thelma and Louise', 'Indiana Jones'). 


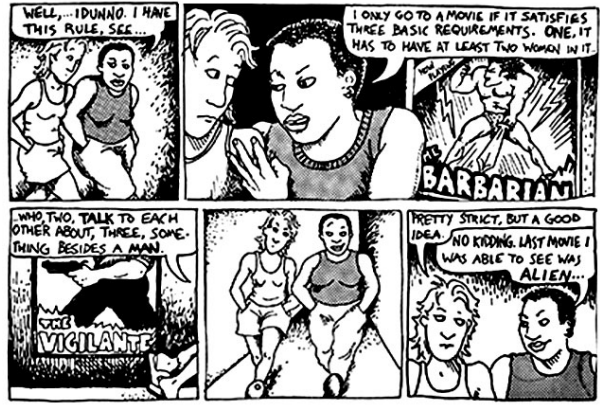

(a) 'Dykes to Watch Out For' by Alison Bechdel, 1985

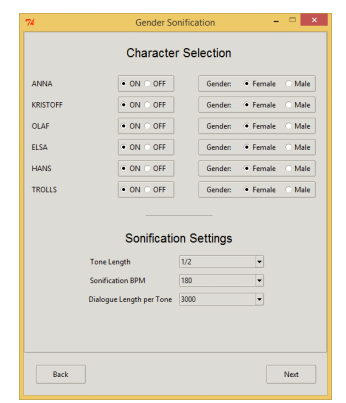

(b) Prototype GUI

Fig. 1: Bechdel test (left) and our gender sonification prototype (right)

An informal presentation of the results provided some interesting insights and the movies were paired to their sonifications with relative ease by the participants. The next step was to make the sonification an automated process. Scripts of various popular movies were collected in the 'Fountain' format - an open-source format for script-writing. We then created a prototype program (see Fig. 1b) that could load the script file, analyze it, present sonification options to a user and then create the audio output in wave format. In the analyzation step of the program, the source script is broken down in dialogue and action segments based on the formatting provided by the 'Fountain' file format. The program creates a list of all movie characters by looking at which names precede a dialogue segment, establishes a total word count per character and presents the six characters with the most lines to the user.

After the analysis, the user chooses whether or not to include each of the six characters, assigns their gender, and adjusts the sonification settings in terms of tone length, beats per minute, and dialogue length per tone. Tone length and beats per minute determine the overall speed of the sonification and can be considered as simply two ways of letting a user set the speed. Dialogue length per tone represents the amount of letters that each tone-segment contains. The default setting of 3000 means that the source script uses 3000 letters per tonesegment and creates tones for each character included within that count. If three characters have a dialogue of 1000 letters within a tone-segment, three notes will sound at the same time, each of them at $1 / 3$ of the full volume.

In the sonification, female characters are represented by a higher pitched sound, and male characters by a lower pitch, as this is the option most likely to be intuitive to the listener [6]. In addition, we differentiated between characters with smaller changes in pitch. In the first prototype, this was done by age, as adult female voices get lower with age while male voices get higher, so this is again a realistic option [7]. In the current prototype, age is not factored in and the pitch within a gender's pitch range is fixed in order of amount of dialogue. 


\subsection{Focus Groups}

Two small focus groups ( $\mathrm{N}=3$ and $\mathrm{N}=4$ ) were organized to test the current prototype and discuss the resulting sonifications. Participants were between 18 and 29 years of age, 6 female, 1 male. The first group was a mix of different people, the second group consisted of Dutch film-students. The groups listened to two audio-files, knowing which movies they were based on but initially not how the sonification worked. After first impressions, they were explained more about the mechanism and the reasons behind the project, and made their own sonifications with the program. They were asked for their first impressions, thought and ideas on the sonifications, opinions on gender representation in movies in general, and wishes as a movie-audience and/or as movie-creators.

\section{Results}

Most of the participants thought the sonification had something to do with the storyline or who the main characters were before they were told what kind of information (gender representation based on amount of dialogue) was sonified. After the sonification parameters were explained, some participants found it difficult to clearly distinguish between individual characters based on their pitch. Several participants noted that some movies sounded more different than expected. The first group remembered ' $\mathrm{V}$ for Vendetta' as a mostly male dominated movie, and were surprised to hear many high tones.

One group noted that the pitch-differences made female-centered movies seem very happy, but male-centered movies sounded like "funeral music". Movies like 'Frozen' and 'Sister Act' sounded more musical, with many tones at once - an effect of many characters having dialogue lines in a given scene.

We found that only some participants described themselves as conscious of gender representation in movies. One participant noted that they might unconsciously notice it. They agreed that there was usually a huge overlap between gender representation and film genre. One participant noted that situations where expectations were not met would be most interesting for sonification, such as "when you know it's an action movie but you hear only high notes".

Most participants noted that, just as with the Bechdel test, the way each gender was depicted in the movie was not translated into the audio-file. Although some regarded this as a loss, most agreed that it would be difficult to introduce this information in an objective manner. Participants were divided on whether the sonification was too abstract to gain meaningful information about the movie. Some thought it was too vague, but one participant noted that it "gave me an understanding that is perhaps more accurate than just data, because representation is not always factual".

Participants noted that the separation of the visual element worked well to experience the movie in a more unbiased manner, also noting that with some additional information brought in, a sonification could act as a spoiler-free trailer.

The first focus group largely agreed on a distinction between the perceived quality of the movie itself in whether gender-representation was important to 
them. One of them noted they "would only care about more ambitious movies, not about the ones that you already know will be stupid". Others agreed that gender-representation was interesting from a research-perspective, but did not much factor into their own private choice of movies. The second group wondered how significant speaking lines really are for judging the importance of a character. They thought the combination of the "associative qualities of sound" and the "hard facts of word-counting" was interesting, but could also be confusing.

\subsection{Recommendations}

Most participants would have liked the sonification to offer more information, particularly on the quality or topic of the conversation, the story-line or the movie genre. Mentioned ways to translate this were in speed, volume, soundorigin and different instruments. Some mentioned that this would also increase the chance that a person less interested in gender issues might want to hear the sonification. Multiple participants were interested in whether movies of the same genre would sound the same, or whether their own favorite movies would. Participants noted that it could be interesting to see how different people sonify different movies, as so many elements are subjective. The second group was interested in sonifying the (short) movie scripts they were currently working on for their studies - these sounded about as they had expected.

\section{Conclusion and Discussion}

From our focus groups we learned that the sonification of gender is not selfexplanatory even if an audience is aware that an aspect of a movie is sonified. As such, it is necessary to make the purpose as well as the mapping clear from the beginning on if the goal is to promote a focused discussion. While gender sonification is essentially a qualitative representation of gender balance in a movie, it has been identified as very data-driven. We think that, due to the subjective nature of what constitutes as balanced representation, an intuitive approach can be useful, but many participants expressed their desire to see more tangible data on this subject. Clearly, gender sonification cannot be a replacement for an in-depth analysis using more traditional forms of reporting data. As such we anticipated that it could be considered unpractical and vague. However, it can also be understood as a playful method to add a experiential component to the representation of data. In our small focus groups we were indeed pleased with the amount of discussion the sonifications evoked. Acting as a starting point for such conversation is, as we consider it, the main function of this form of data representation.

Arguably, this effect is harder to achieve outside of a dedicated focus-group environment - meaning that the system must promote and facilitate such discussions. One possible way to do this is through letting users compare their expectations with the reality, another to promote a social experience were multiple users share their thoughts about a sonification. A movie/sonification pairing 
game, as was done with our first prototype, could also bring unexpected results to light and facilitate discussion.

A downside of our mapping scheme is that we largely lose the rhythm of the movie, since we mainly focus on text (scripts have some descriptions of actions but this does not translate well to screen-time). Since we focus specifically on gender representation, however, we consider this an acceptable loss.

Adding more information to the audio can be useful for pointing out unanticipated combinations of elements - like a genre associated with a certain gender that turns out to feature mostly characters from another gender. This has the additional benefit of being able to inform on representation of different sexualities, for instance by pointing out there is a love scene featuring only male characters. Additionally, it might get people interested for whom just gender representation is not an interesting enough factor when choosing or evaluating a movie. This can mean focusing on the story-line or genre, but also to incorporate the representation of other minority groups. One obvious example would be characters of a non-binary gender, who are currently largely ignored not only in the film industry, but also in our prototypes so far.

We think the strength of this representation is to be found in the balance between simplicity and narrative power. There is no important other information present to distract from the main point of the audio, thus drawing attention to an issue that might have escaped attention when watching the actual movie. At the same time, it is still interesting and organic, as it changes over time, different characters can be heard and a little bit of the flow of the movie is discernible. It does not give clear-cut answers on what the gender-representation is or how it should be, leaving room for different opinions and their exchange.

\section{References}

1. Bleakley, A., Jamieson, P.E., Romer, D.: Trends of sexual and violent content by gender in top-grossing us films, 1950-2006. Journal of Adolescent Health 51(1) (2012) 73-79

2. Smith, S.L., Choueiti, M., Scofield, E., Pieper, K.: Gender inequality in 500 popular films: Examining on-screen portrayals and behind-the-scenes employment patterns in motion pictures released between 2007-2012. Study by the University of Southern California Annenberg School for Communication \& Journalism (2013)

3. Website, B.: Bechdel test movie list (2015) [Online; accessed 28-April-2015].

4. Peterson, P.: Beyond the bechdel test: Two (new) ways of looking at movies (2014) [Online; accessed 28-April-2015].

5. Walker, B.N., Cothran, J.T.: Sonification sandbox: A graphical toolkit for auditory graphs. (2003)

6. Titze, I.R.: Physiologic and acoustic differences between male and female voices. The Journal of the Acoustical Society of America 85(4) (1989) 1699-1707

7. Honjo, I., Isshiki, N.: Laryngoscopic and voice characteristics of aged persons. Archives of Otolaryngology 106(3) (1980) 149-150 